Research Paper

\title{
CD44+/CD105+ Human Amniotic Fluid Mesenchymal Stem Cells Survive and Proliferate in the Ovary Long-Term in a Mouse Model of Chemothera- py-Induced Premature 0varian Failure
}

\author{
Te Liu'1,2,5凶 , Yongyi Huang ${ }^{5}$, Lihe Guo ${ }^{3,5}$, Weiwei Cheng ${ }^{2}$, Gang Zou ${ }^{4}$
}

1. Shanghai Geriatric Institute of Chinese Medicine, Longhua Hospital, Shanghai University of Traditional Chinese Medicine, Shanghai 200031, China;

2. International Peace Maternity and Child Health Hospital, Shanghai Jiaotong University, Shanghai 200030, China;

3. Institute of Biochemistry and Cell Biology, Shanghai Institute for Biological Sciences, Chinese Academy of Sciences, Shanghai 200031, China;

4. Shanghai First Maternity and Infant Hospital, Tongji University School of Medicine, Shanghai 200040, China;

5. Sino-America United Stem Cell Research Center, Shanghai 200333, China.

Corresponding author: Te Liu Ph.D., Shanghai Geriatric Institute of Chinese Medicine, Longhua Hospital, Shanghai University of Traditional Chinese Medicine, Shanghai 200031, P.R. China, Phone: 86-21-64720010; Fax: 86-21-64720010; E-Mail: liute79@yahoo.com.

(C) Ivyspring International Publisher. This is an open-access article distributed under the terms of the Creative Commons License (http://creativecommons.org/ licenses/by-nc-nd/3.0/). Reproduction is permitted for personal, noncommercial use, provided that the article is in whole, unmodified, and properly cited.

Received: 2012.07.09; Accepted: 2012.09.04; Published: 2012.09.11

\begin{abstract}
Objectives: Stem cell transplantation has been reported to rescue ovarian function in a preclinical mouse model of chemotherapy-induced premature ovarian failure (POF); however, maintaining the survival and self-renewal of transplanted seed cells in ovarian tissues over the long-term remains a troublesome issue. In this study we aimed to determine whether the CD44+/CD 105+ human amniotic fluid cell (HuAFCs) subpopulation represent potential seed cells for stem cell transplantation treatments in POF. Materials and methods: The CD44+/CDI05+ subpopulation were isolated from HuAFCs, cultured in vitro, and injected into a cyclophosphamide-induced mouse model of POF. Results: Under continuous subculture in vitro, $C D 44+/ C D 105+$ cells proliferated rapidly and expressed high levels of the proliferative markers Ki67 and survivin, as well as high levels of a number of mesenchymal stem cell biomarkers. Moreover, when red fluorescence protein (RFP)-transduced CD44+/CD 105+ HuAFCs were transplanted into the ovaries of POF mice, the cells could be detected by fluorescence microscopy up to three weeks after injection. Furthermore, the BrdUrd incorporation assay and immunofluorescent staining demonstrated that CD44+/CDI05+ HuAFCs underwent normal cycles of cell proliferation and self-renewal in the ovarian tissues of POF mice over the long-term. Conclusions: The mesenchymal stem cell properties and long-term in vivo survival of CD44+/CDI05+ HuAFCs make them ideal seed cells for stem cell transplantation to treat POF.
\end{abstract}

Key words: Mouse premature ovarian failure model; cell transplantation; human amniotic fluid cells; mesenchymal stem cell like cells; long-term survival; proliferation.

\section{Introduction}

Increasing attention is being paid to premature ovarian failure (POF), which is one of the most threatening diseases to female reproductive health. Approximately $1-5 \%$ of women worldwide experi- 
ence cessation of their menstrual cycle prior to age 40, a condition known as POF [1-8]. Patients with POF have a number of typical characteristics $[6,7]$ : (a) primary or secondary amenorrhea; (b) at least intermittent hypoestrogenism; (c) hypergonadotropinism; and (d) age under 40 years at the time of onset. In some patients with POF, laparoscopy reveals a lack of developing follicles and ovarian biopsy shows a network of connective tissue interspersed with fibroblasts. The uterus and vaginal mucosa undergo atrophy in patients with POF, due to a lack of estrogen stimulation from the inactive ovaries $[2,3]$. The incidence of POF has increased in recent years. Although some treatments are currently available, the condition cannot be reversed, and it would be desirable to find improved therapeutic strategies for POF.

Human amniotic fluid cells (HuAFCs) are currently used for the routine prenatal genetic diagnosis of a wide range of foetal abnormalities $[9,10]$. However, amniotic fluid contains multiple types of cells derived from the developing foetus. These cells can differentiate into a variety of cell types, including adipose, muscle, bone and neuronal cells $[11,12]$. The CD44+/CD105+ subpopulation can be directly sorted from human amniotic fluid and cultured for ex vivo expansion [13-17]. Previous studies have demonstrated the ability of CD44+/CD105+ cells to differentiate into ectodermal, endodermal, mesodermal, hepatic cells and cardiac muscle cells [14]; therefore, the CD44+/CD105+ human amniotic fluid subpopulation can differentiate into all three germ layers. Additionally, our previous studies indicated that HuAFCs express a variety of growth factors, including epidermal growth factor (EGF), basic fibroblast growth factor (bFGF), transforming growth factor alpha (TGF- $\alpha$ ), transforming growth factor beta (TGF- $\beta$ ) and bone morphogenetic protein 4 (BMP-4), as well as the stem cell markers Nanog, Oct4 and Nestin. More importantly, HuAFCs lack major histocompatibility complex class II antigens and express only low levels of major histocompatibility complex class I antigens [18-21]. Indeed, we have reported that dopaminergic neuron-like cells derived from CD44+ HuAFCs were ameliorative to behavioural recovery in a rat model of Parkinson's disease, and also that CD44+/CD105+ HuAFCs could be induced to differentiate into pancreatic $\beta$-cell-like cells [19-21]. HuAFCs cells are more easily accessible than other adult stem cells, making them a potential autologous donor source for stem cell therapy. Therefore, in this study we aimed to determine which subpopulations of HuAFCs can survive and proliferate in vitro and in vivo, and investigated whether the CD44+/CD105+ HuAFC subpopulation have the properties of mes- enchymal stem cells and represent potential seed cells for stem cell transplantation to treat POF.

\section{Materials and Methods}

\section{Isolation and in vitro expansion of CD44 and CDI05 phenotype cells by magnetic activated cell sorting system}

Human amniotic fluids were obtained by ultrasound-guided amniocentesis performed on pregnant women for routine prenatal diagnosis purposes at gestational ages ranging from $18^{\text {th }}-22^{\text {th }}$ weeks. All of the 10 human samples were obtained after approval from the Ethical Review Board of the Shanghai First Maternity and Infant Hospital (Shanghai, China) and after obtaining written informed consent from subjects. CD44+/CD105+ subpopulation cells were isolated from those human amniotic fluid using $4 \mu \mathrm{l}$ of the primary monoclonal antibodies (mouse anti-human CD44-FITC, rabbit anti-human CD105-PE, eBioscience Inc, San Diego, CA, USA)) stored at $4^{\circ} \mathrm{C}$ in PBS for $30 \mathrm{~min}$ in a volume of $1 \mathrm{ml}$ as previously described [17-21]. After reaction, the cells were washed twice in PBS, and were put the secondary monoclonal antibodies (Goat anti-mouse or Goat anti-rabbit coupled to magnetic microbeads, Miltenyi Biotec, Auburn, CA), incubated at $10^{\circ} \mathrm{C}$ in PBS for $15 \mathrm{~min}$ and then washed twice in PBS. The subpopulation cells were plated at $1 \times 10^{6}$ cells $/ \mathrm{ml}$ in DMEM:F12 (1:1) (Gibco, Gaithersburg, MD, USA), supplemented with $10 \mathrm{ng} / \mathrm{ml}$ basic fibroblast growth factor (bFGF), $10 \mathrm{ng} / \mathrm{ml}$ epidermal growth factor (EGF) (all from Sigma-Aldrich, St Louis, USA), 10\% fetal bovine serum and $2 \mathrm{mM}$ L-glutamine (all from Gibco, Gaithersburg, MD, USA). All CD $44^{+} \mathrm{CD} 105^{+}$cells were cultured in a humidified incubator, at $37^{\circ} \mathrm{C}$ with $5 \% \mathrm{CO}_{2}$, until $80 \%$ confluent. In this experiment, all cells had been cultured on the same conditions until passage $4^{\text {th }}$ before making ulterior experiments.

\section{MTT assay for cell proliferation}

Each group of cells was seeded at $2 \times 10^{3}$ cells per well in 96-well plates, and cultured in DMEM:F12 (1:1) supplemented with $10 \%$ fetal bovine serum at 37 ${ }^{\circ} \mathrm{C}$ with $5 \% \mathrm{CO}_{2}$, until $85 \%$ confluence had been reached. MTT (Sigma-Aldrich, St Louis, USA) (5 $\mathrm{mg} / \mathrm{mL}$ ) was added to the maintenance cell medium at different time points, and incubated at $37{ }^{\circ} \mathrm{C}$ for an additional $4 \mathrm{~h}$. The reaction was terminated with 150 $\mu \mathrm{L}$ of dimethylsulfoxide (Sigma-Aldrich, St Louis, USA) per well, the cells were lysed for $15 \mathrm{~min}$, and the plates were gently shaken for $5 \mathrm{~min}$. Absorbance values were determined with an ELISA reader (Model 680; Bio-Rad, Hercules, CA, USA) at $490 \mathrm{~nm}$. 


\section{RNA extraction and analysis by quantitative real-time PCR}

Total RNA from each cell was isolated with Trizol Reagent (Invitrogen, Life Technologies Corporation, Grand Island, NY, USA), according to the manufacturer's protocol. The RNA samples were treated with DNase I (Sigma-Aldrich, St Louis, USA), quantified, and reverse-transcribed into cDNA with the ReverTra Ace- First Strand cDNA Synthesis Kit (TOYOBO, TOYOBO (SHANGHAI) BIOTECH CO., LTD., Shanghai, China). Quantitative real-time PCR was conducted with a RealPlex4 real-time PCR detection system from Eppendorf (Germany), with SyBR Green RealTime PCR Master Mix (TOYOBO, TOYOBO (SHANGHAI) BIOTECH CO., LTD., Shanghai, China) as the detection dye. Quantitative real-time PCR amplification was performed over 40 cycles with denaturation at $95{ }^{\circ} \mathrm{C}$ for $15 \mathrm{~s}$ and annealing at $58^{\circ} \mathrm{C}$ for $45 \mathrm{~s}$. Target cDNA was quantified with the relative quantification method. A comparative threshold cycle $(\mathrm{Ct})$ was used to determine gene expression relative to a control (calibrator), and steady-state mRNA levels are reported as an n-fold difference relative to the calibrator. For each sample, the maker gene $\mathrm{Ct}$ values were normalized with the formula $\Delta \mathrm{Ct}=\mathrm{Ct}$-genes $-\mathrm{Ct} \_18 \mathrm{~S}$ RNA. To determine relative expression levels, the following formula was used: $\Delta \Delta \mathrm{Ct}=\Delta \mathrm{Ct}$ all_groups $-\Delta \mathrm{Ct} \_$blankcontrol_ group. The values used to plot relative expressions of markers were calculated with the expression $2-\Delta \Delta \mathrm{Ct}$. The mRNA levels were calibrated on the basis of levels of 185 rRNA. The cDNA of each gene was amplified with primers as previously described: Ki67-FP: TGGGTCTGTTATTGATGAGCC; TGACTTCCTTCCATTCTGAAGAC; TTGGTGAATTTTTGAAACTGGA; CTTTCTCCGCAGTTTCCTCA; CGTTGATTAAGTCCCTGCCCTT; TCAAGTTCGACCGTCTTCTCAG.

\section{Western blotting analysis}

Cells were lysed using a $2 \times$ loading lysis buffer (50 mM Tris- $\mathrm{HCl}, \mathrm{pH} 6.8,2 \%$ sodium dodecyl sulfate, $10 \% \beta$-mercaptoethanol, $10 \%$ glycerol and $0.002 \%$ bromophenol blue). The total amount of proteins from the cultured cells was subjected to $12 \%$ SDS-PAGE and transferred onto Hybrid-PVDF membrane (Millipore, Bedford, USA) membranes. After blocking with $5 \%(\mathrm{w} / \mathrm{v})$ non-fat dried milk in TBST (Tris-buffered saline containing Tween-20) (25 mM Tris/ $\mathrm{HCl}, \mathrm{pH} 8.0,125 \mathrm{mM} \mathrm{NaCl}$ and $0.05 \%$ Tween-20), the PVDF membranes were washed 4 times (15 min each) with TBST at room temperature and incubated with primary antibody (rabbit an- ti-human Ki67 polyclonal antibody (1:1000; Chemicon, Temecula, CA, USA) and rabbit anti-human Survivin polyclonal antibody (1:1000; Chemicon, Temecula, CA, USA)). Following extensive washing, membranes were incubated with HRP-conjugated goat anti-rabbit IgG secondary antibody (1:1000; Santa Cruz Technology, Santa Cruz, USA) for $1 \mathrm{~h}$. After washing 4 times (15 min each) with TBST at room temperature, the immunoreactivity was visualized by enhanced chemiluminescence using ECL kit from Perkin-Elmer Life Science (Norwalk, USA).

\section{Immunofluorescence staining}

The cultured cells were washed 3 times with PBS and fixed with $4 \%$ paraformaldehyde (Sigma-Aldrich, St. Louis, USA) for $30 \mathrm{~min}$. After blocking, the cells were incubated first with rabbit anti-human Ki67 polyclonal antibody (1:200; Chemicon, Temecula, CA, USA) and rabbit anti-BrdU polyclonal antibody (1:200; Sigma-Aldrich, St Louis, USA) overnight at 4 ${ }^{\circ} \mathrm{C}$, and then with FITC-conjugated goat anti-rabbit IgG antibody (1:200; Sigma-Aldrich, St Louis, USA) and $5 \mu \mathrm{g} / \mathrm{ml}$ DAPI (Sigma-Aldrich, St Louis, USA) at room temperature for $30 \mathrm{~min}$. Then the cells were thoroughly washed with TBST and viewed through a fluorescence microscope (DMI3000; Leica, Allendale, NJ, USA).

\section{The mouse model of premature ovarian failure and xenografing experiments}

Hebetic femal C57BL/ 6 mice $(n=12)$, between 4 and 5 weeks of age, obtained from the Shanghia Tongii University with Institutional Anminal Care and Use Committer approval in accordance with institutional guidelines. All mice were maintained for 14 days, 3 to 4 per cage, in a temperature controlled coloney room under standard light-dark cycle with free access to food and water. The study protocol was according the manuscript [4] and had some ameliorations. To build the POF model, mice were administered single intraperitoneal injection of $70 \mathrm{mg} / \mathrm{kg}$ cyclophosphamide (Sigma-Aldrich, St Louis, USA) at 6 weeks of age. The animals were divided into 2 groups: a negative control groups ( 6 animals of POF model) grafted with CD44-/CD105- HuAFCs in right ovarian, and an experimental group ( 6 animals of POF model) grafted with CD44+/CD105+ HuAFCs in in right ovarian. One weeks after mouse POF model built successfully, each experimental model received an injection of $10 \mu 1$ of HuAFCs (approximately $1 \times 10^{3}$ cell spheres $/ \mu \mathrm{l}$ ), which was harbored a red fluorescence

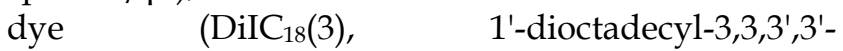
tetramethylindocarbocyanine perchlorate), or PBS. 
The ulterior experiments of the animals model in both groups on 7, 14 and 21 day after transplantation.

\section{ELISA assay}

The mouse estradiol $\left(\mathrm{E}_{2}\right)$ and follicle stimulating hormone (FSH) ELISA kit (Westang Bio, Shanghai, China) was used according to the protocol description to determin the level of $\mathrm{E}_{2}$ or FSH in mouse plasma. Briefly, $100 \mu \mathrm{l}$ of mouse $\mathrm{E}_{2}$ or FSH standards at concentrations of $0,50,300,1000$ and $5000 \mathrm{pg} / \mathrm{ml}$ or 0,5 , $10,25,50$ and $100 \mathrm{mIU} / \mathrm{ml}$ or diluted mouse plasma were added to anti- $\mathrm{E}_{2}$ or $\mathrm{FSH}$ antibody precoated microtest wells and incubated for $60 \mathrm{~min}$. After 3 times wash, the HRP-conjugated detection antibodies were then added followed by addition of the substrate solution. The absorbance was determined at a wavelength of $450 \mathrm{~nm}$.

\section{Flow cytometric (FCM) analysis of cell cycle by PI staining.}

Each group cells was seeded at $3 \times 10^{5}$ per well in 6-well plates and cultrued until $85 \%$ confluent. Each group cells was washed by PBS on three times, then were collected by centifugation (Allegra X-22R, Beckman Coulter) at $1000 \mathrm{~g}$ for $5 \mathrm{~min}$. The cell pellets were the resuspended in $1 \mathrm{~mL}$ of PBS, fixed in $70 \%$ ice-cold ethanol, and ketp in a freezer more than $48 \mathrm{~h}$. Before flow cytometric analysis, The fixed cells were centrifuged, washed twice with PBS, and resuspended in PI staining solution (Sigma Chemicals) containing $50 \mu \mathrm{L} / \mathrm{mL}$ PI and $250 \mu \mathrm{g} / \mathrm{mL}$ RNase A (Sigma Chemicals). The cell suspension, which was hidden from light, were incubated for $30 \mathrm{~min}$ at $4^{\circ} \mathrm{C}$ and analyzed using the FACS (FCM-500, Beckman Coulter). A total of 10,000 events were acquired for analysis using CellQuest software.

\section{Statistical analysis}

Each experiment was performed as least three times, and data are shown as the mean \pm standard error where applicable; differences were evaluated with Student's t-tests. A $p$-value of $<0.05$ was considered to be statistically significant.

\section{Results}

\section{Isolation and enrichment of the CD44+/CD I 05+ subpopulation from human amniotic fluid}

Multiple cell types can be isolated from human amniotic fluid. Microscopy revealed that various different colonies formed after HuAFCs were cultured for 10 days in vitro (Figure 1). Previous studies have suggested that the CD44 and CD105-expressing cell subpopulation in human amniotic fluid is relatively small. Therefore, we used a magnetic activated cell sorting system to isolate and enrich this specific subpopulation. The cells were detected by flow cytometry (FCM), and CD44+/CD105+ cells represented 1.75\% \pm $0.25 \%$ of HuAFCs (Figure 1), indicating that CD44+/CD105+ cells could be successfully enriched from human amniotic fluid using magnetic activated cell sorting. Moreover, the CD44+/CD105+ HuAFCs had remarkable morphological differences compared to the remaining HuAFC subpopulation. CD44+/CD105+ HuAFCs had a typical mesenchymal morphology; whereas CD44-/CD105- HuAFCs were spindle-shaped or polygonal, and retained a cobblestone appearance (Figure 1).

\section{CD44+/CD I05+ HuAFCs proliferate rapidly and express high levels of mesenchymal stem cell biomarkers}

The MTT assay was used to determine the viability of CD44+/CD105+ and CD44-/CD105$\mathrm{HuAFCs}$ at the same passage over time in vitro. There was no significant difference in the viability of CD44+/CD105+ HuAFCs and CD44-/CD105$\mathrm{HuAFCs}$ at $24 \mathrm{~h}$ after isolation; however, the viability of CD44-/CD105- HuAFCs significantly reduced at 2 $\mathrm{d}$ and $5 \mathrm{~d}$ compared to CD44+/CD105+ HuAFCs (Figure 2), indicating that CD44+/CD105+ HuAFCs proliferated more rapidly than CD44-/CD105HuAFCs (Table 1). FCM indicated that CD44-/CD105- HuAFCs were arrested in the G2/M phase of the cell cycle, with a significantly reduced percentage of $S$ phase cells (Table 2); whereas CD44+/CD105+ HuAFCs displayed a normal cell cycle distribution and underwent cell division (Figure 2 ). To confirm that CD44+/CD105+ HuAFCs proliferated in vitro, the expression of proliferation-related markers were assayed by quantitative real-time polymerase chain reaction (qRT-PCR) and Western blotting. The relative expression levels of several important proliferation-related markers were compared using qRT-PCR, and normalized to the internal control 18S rRNA. Ki67 and survivin mRNA were expressed at significantly higher levels in CD44+/CD105+ HuAFCs than CD44-/CD105HuAFCs (Figure 2). Western blotting confirmed that Ki67 and survivin were expressed at significantly higher levels in CD44+/CD105+ HuAFCs (1.000 \pm 0.056 and $1.000 \pm 0.035)$ than CD44-/CD105- HuAFCs $(0.229 \pm 0.054$ and $0.339 \pm 0.049$ relative to GAPDH levels, respectively; Figure 2).

In order to evaluate the degree of mesenchymal stem cell "stemness" in the CD44+/CD105+ and CD44-/CD105- HuAFC subpopulations, we analyzed 
the expression of several mesenchymal stem cell biomarkers using FCM (Figure 2). Expression of the "stemness" markers CD29, CD44, CD73, CD90, CD105 and CD166 was approximately 60- to 90 -fold higher in CD44+/CD105+ HuAFCs than CD44-/CD105HuAFCs, suggesting that the CD44+/CD105+ subpopulation possess more of the properties of mesenchymal stem cells than the CD44-/CD105- subpopulation.

\section{Establishment of a mouse model of chemo- therapy-induced premature ovarian failure}

In order to evaluate whether the mouse POF model was established successfully, the plasma $\mathrm{E}_{2}$ and FSH levels and ovarian pathology were investigated. After injection of cyclophosphamide, the plasma $E_{2}$ and FSH levels reduced over time in the POF mouse model group, but not in the normal health mouse. ELISA assays indicated that the POF mouse models lacked hormonal maintenance from the ovaries (Figure 3). In addition, ovarian pathology revealed that the ovaries of the health normal health mouse ontained a large number of follicles at all stages of immaturity or maturation (Figure 3). In contrast, the atrophied ovaries of the POF model mice were mostly composed of interstitial cells in a fibrous matrix, with a reduced number of follicles at each stage. Additionally, the ovaries of the POF model mice contained an increased number of collapsed oocytes (Figure 3).

Table I. MTT assay determination of the viability of HuAFCs in vitro

\begin{tabular}{lll}
\hline Day & $\begin{array}{l}\text { CD44+/CD105+ HuAFCs (n } \\
=3)\end{array}$ & $\begin{array}{l}\text { CD44-/CD105- HuAFCs (n } \\
=3)\end{array}$ \\
\hline 0 & $1.67 \pm 0.88$ & $1.48 \pm 0.68$ \\
1 & $3.80 \pm 2.91$ & $2.56 \pm 1.15$ \\
2 & $13.73 \pm 2.54$ & $4.45 \pm 2.13$ \\
3 & $38.73 \pm 3.54$ & $15.02 \pm 4.69$ \\
4 & $56.95 \pm 6.70$ & $27.44 \pm 1.58$ \\
5 & $65.24 \pm 2.39$ & $38.09 \pm 1.91$ \\
\hline
\end{tabular}

Table 2. Mesenchymal stem cell biomarkers expression of HuAFCs determined by FCM

\begin{tabular}{lllllll}
\hline Cells & $\begin{array}{l}\text { CD29 } \\
(\%)\end{array}$ & $\begin{array}{l}\text { CD44 } \\
(\%)\end{array}$ & $\begin{array}{l}\text { CD73 } \\
(\%)\end{array}$ & $\begin{array}{l}\text { CD90 } \\
(\%)\end{array}$ & $\begin{array}{l}\text { CD105 } \\
(\%)\end{array}$ & $\begin{array}{l}\text { CD166 } \\
(\%)\end{array}$ \\
\hline CD44-/CD1 & $0.25 \pm 0$. & $0.15 \pm 0.0$ & $1.15 \pm 0$. & $0.25 \pm 0$. & $0.20 \pm 0$. & $0.15 \pm 0$. \\
$05-$ & 05 & 5 & 05 & 05 & 11 & 05 \\
CD44+/CD & $89.95 \pm 0$ & $83.05 \pm 0$. & $88.70 \pm 0$ & $89.00 \pm 1$ & $65.00 \pm 3$ & $90.45 \pm 0$ \\
$105+$ & .55 & 049 & .32 & .42 & .69 & .52 \\
\hline
\end{tabular}

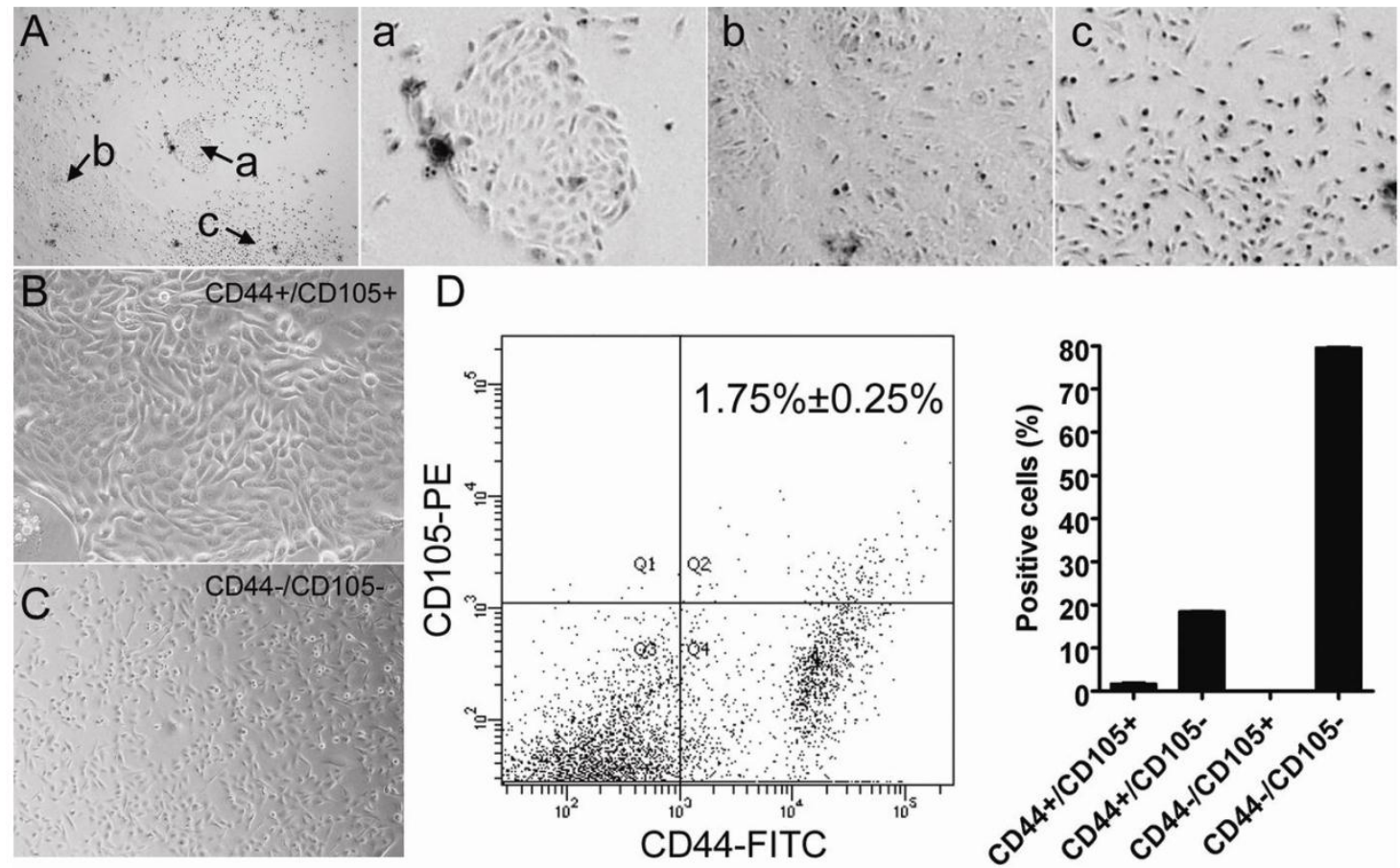

Figure I. Isolation and characterisation of the different subpopulations of cells in human amniotic fluid. (A) Multiple cell populations exist in human amniotic fluid. (a), (b) and (c) illustrate the morphology of different cell subpopulations in human primary amniotic fluid. Original magnification, 200x. (B) Morphology of the CD44+/CD105+ subpopulation isolated from human primary amniotic fluid. Original magnification, 200x. (C) Morphology of the CD44-/CD I05- subpopulation isolated from human primary amniotic fluid. Original magnification, 200x. (D) Isolation of CD44+/CD 105+ HuAFCs from amniotic fluid. The cells were detected by FCM; CD44+/CDI05+ cells represented $1.75 \% \pm 0.25 \%$ of the HuAFC population. 


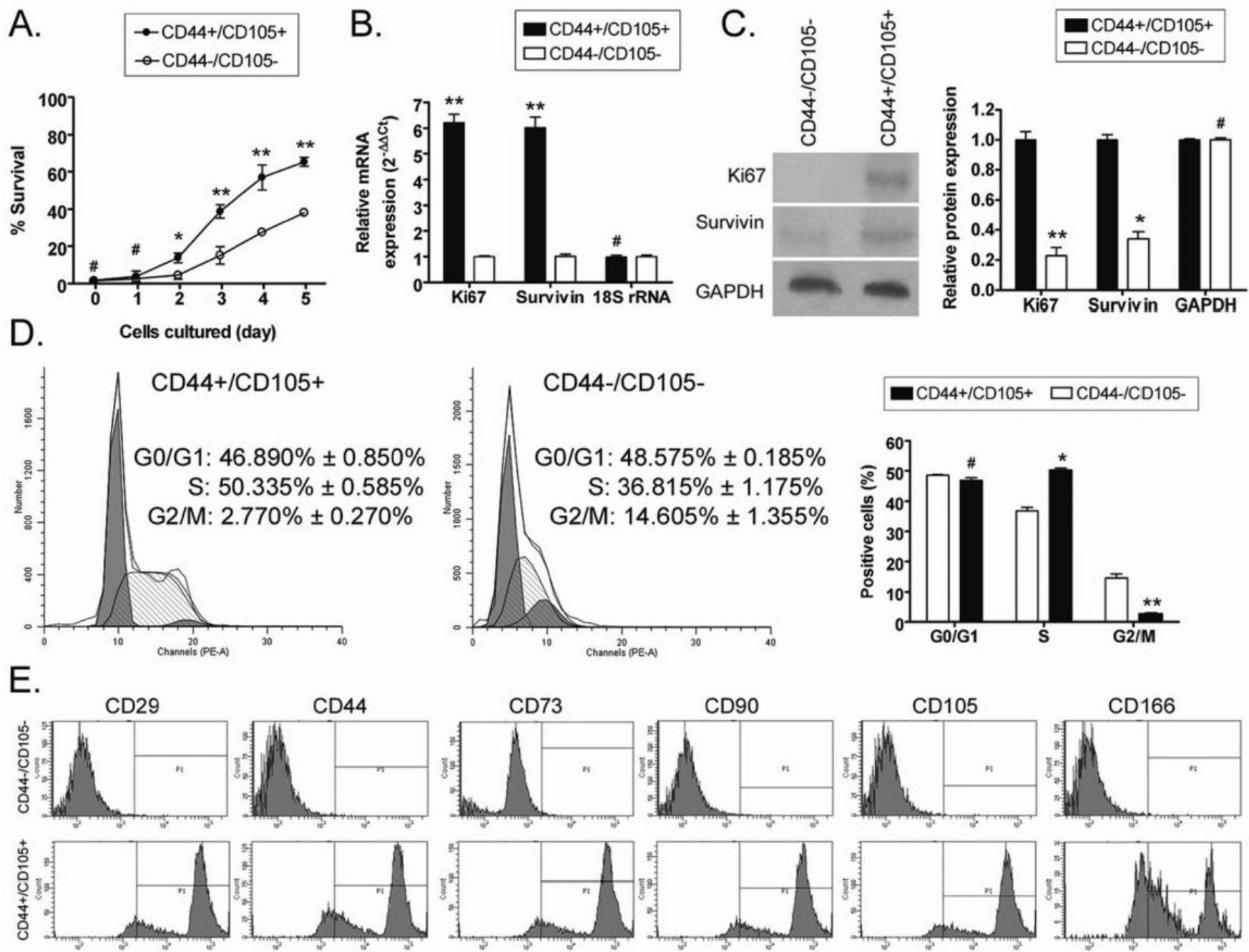

Figure 2. Analysis of the proliferation and survival of CD44+/CD 105+ HuAFCs in vitro. (A) MTT assays indicated that the viability of CD44-/CD I05- HuAFCs significantly reduced at both $2 \mathrm{~d}$ and $5 \mathrm{~d}$, compared to CD44+/CDI05+ HuAFCs. **P<0.01 vs. CD44-/CDI05HuAFCs; *P< 0.05 vs. CD44-/CDI05- HuAFCs; \#P> 0.05 vs. CD44-/CDI05- HuAFCs; $n=3$. (B) qRT-PCR analysis of Ki67 and survivin mRNA expression in CD44+/CD 105+ and CD44-/CDI05- HuAFCs; *P< 0.05 vs. CD44-/CDI05- HuAFCs; \#P>0.05 vs. CD44-/CDI05HuAFCs; $n=3$. (C) Western blotting analysis of Ki67 and survivin protein expression in CD44+/CDI05+ and CD44-/CDI05- HuAFCs; $* * P<0.01$ vs. CD44+/CDI 05+ HuAFCs; $* P<0.05$ vs. CD44+/CDI05+ HuAFCs; \#P> 0.05 vs. CD44+/CDI05+ HuAFCs; $\mathrm{n}=3$. (D) Flow cytometric cell cycle analysis of CD44+/CD 105+ and CD44-/CDI05- HuAFCs. The majority of CD44-/CDI05- HuAFCs were arrested in the G2/M phase with a reduced percentage of $S$ phase cells; **P<0.01 vs. CD44+/CDI05+ HuAFCs; $* P<0.05$ vs. CD44+/CDI05+ HuAFCs; \#P > 0.05 vs. CD44+/CDI05+ HuAFCs; $n=3$. (E) FCM analysis of human mesenchymal stem cell marker expression in CD44+/CD 105+ and CD44-/CD 105- HuAFCs in vitro. Expression of the "stemness" markers was higher in CD44+/CDI05+ HuAFCs than CD44-/CDI05- HuAFCs.

\section{CD44+/CD I 05+ HuAFCs survive and prolifer- ate in the ovaries of the POF model mice}

Red fluorescent protein (RFP)-expressing HuAFCs were injected in vivo and the ovary injection tracts were examined after one, two and three weeks to confirm the presence of transplanted HuAFCs. As anticipated, RFP-positive CD44+/CD105+ HuAFCs could be observed along the injection tract after one, two and three weeks in the ovaries of mice from the
POF model (Figure 4), demonstrating that the transplanted CD44+/CD105+ HuAFCs could survive within the POF mouse ovaries for at least three weeks in vivo. and morphological change can be seen after that. RFP-positive CD44-/CD105- HuAFCs could not be found along the injection tract in the ovaries of mice from the POF model, confirming that only grafted CD44+/CD105+ HuAFCs survived in vivo.

In addition, analysis of cell proliferation using FCM revealed that the number of CD44+/CD105+ 
HuAFCs elevated appreciably in vivo from the time of the graft to three weeks after transplantation (Figure 5), whereas RFP-transduced CD44-/CD105- HuAFCs could not be detected by FCM. Furthermore, BrdUrd incorporation and IF staining revealed that transplanted CD44+/CD105+ HuAFCs had a normal cell cycle distribution and underwent cell division in vivo (Figure 5).
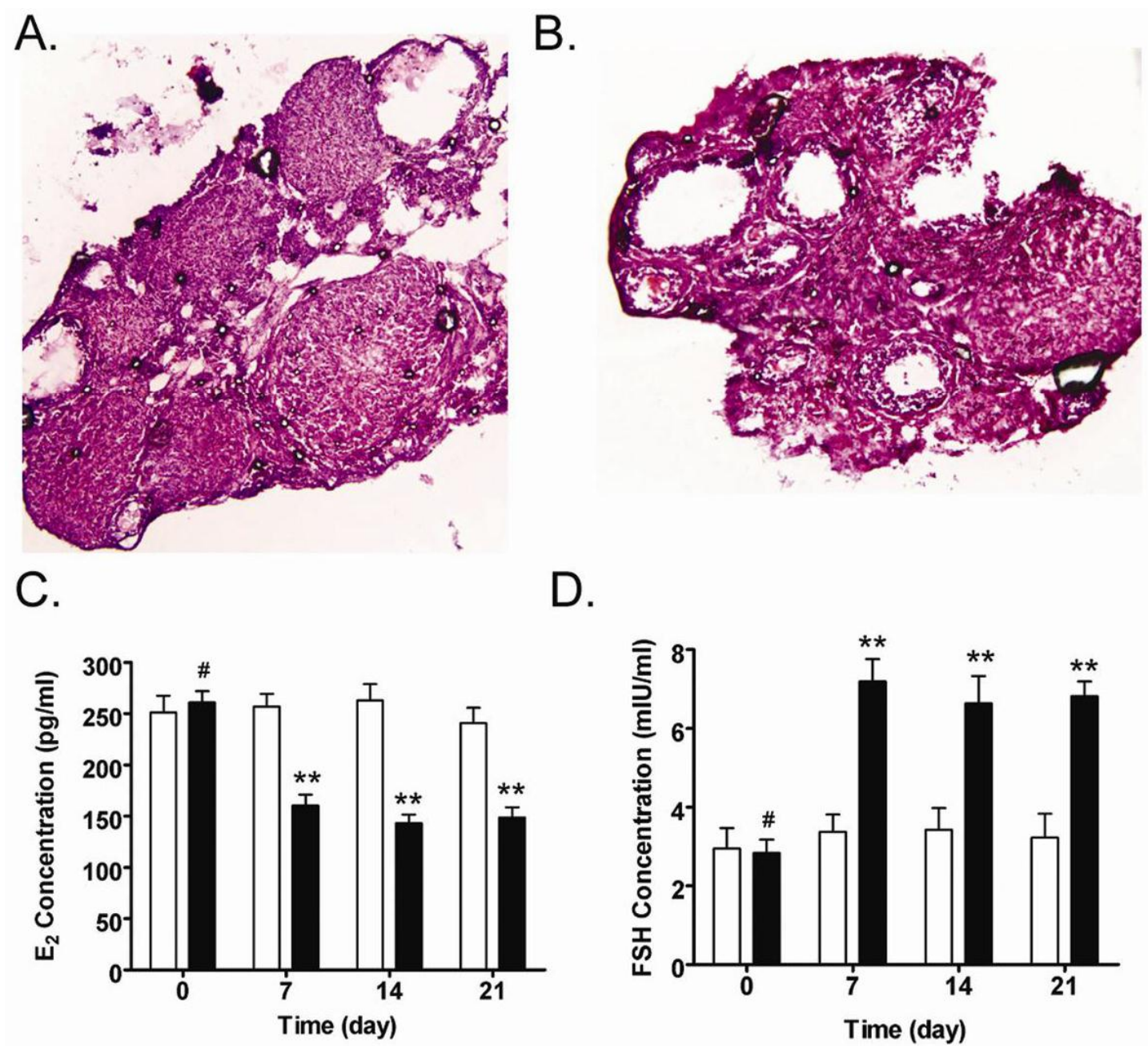

\section{WT POF}

Figure 3. Establishment of a mouse model of POF. (A, B) Ovarian pathology of the wt group (A) and POF group (B) at 2 weeks after injection of cyclophosphamide. The ovaries of the wt group and vehicle group contained a large number of follicles at all stages of immaturity or maturation; whereas the atrophied ovaries of the POF model mice were mostly composed of interstitial cells in a fibrous matrix, with a reduced number of follicles at each stage and an increased number of collapsed oocytes. Original magnification, I00x. (C, D) Plasma $E_{2}$ levels (C) and FSH levels (D) as determined by ELISA at various time points after the injection of cyclophosphamide; **P < 0.01 vs. WT; \#P> 0.05 vs. WT. 


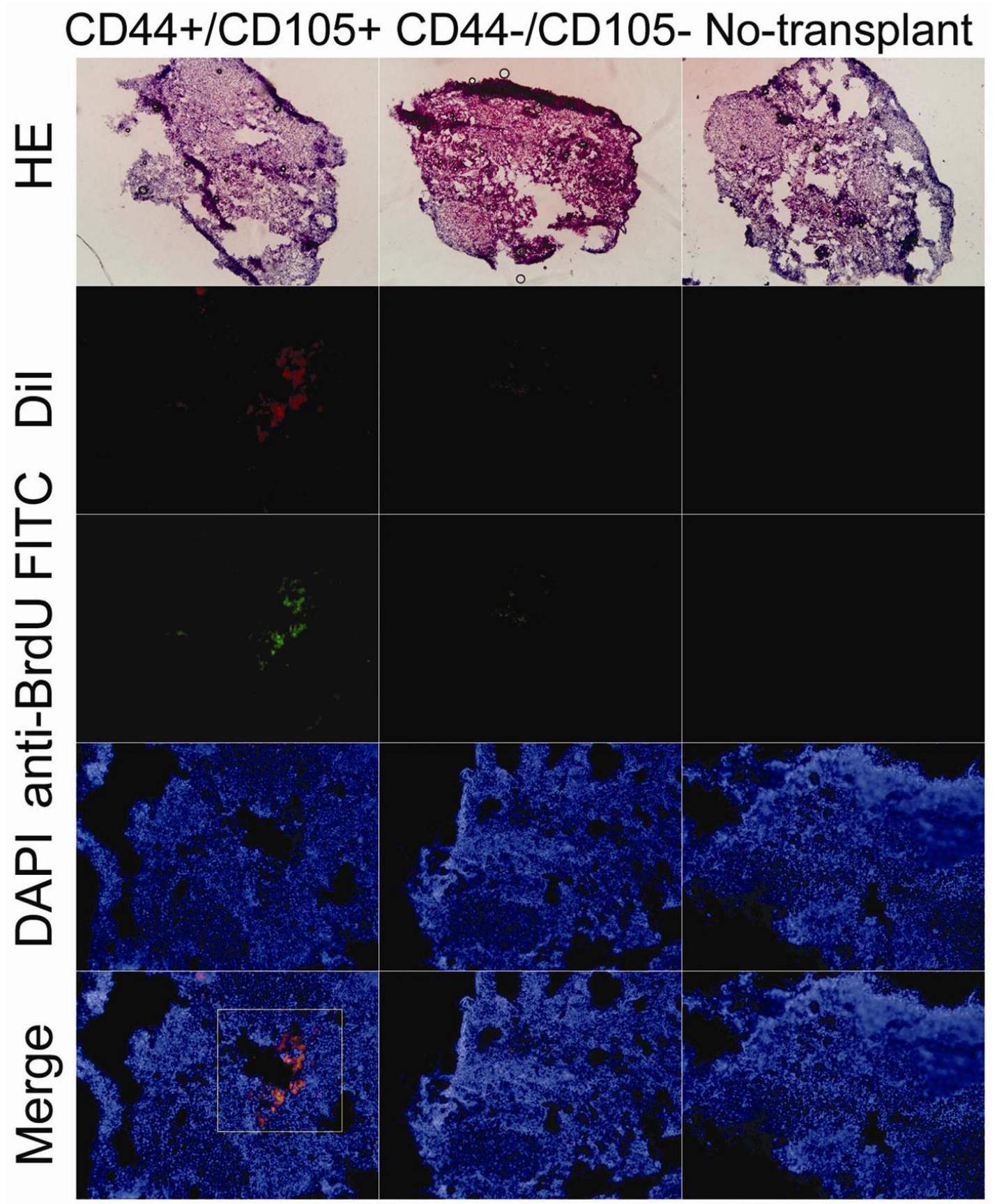

Figure 4. HuAFCs survived in vivo in the ovary in a mouse model of POF. Immunofluorescence (IF) demonstrated that Dil, a marker of HuAFCs, was expressed at high levels in grafted CD44+/CDI05+ HuAFCs transplanted into POF mouse ovaries; the transplanted cells survived for at least three weeks in vivo. In addition, BrdUrd incorporation and IF staining indicated that transplanted CD44+/CDI05+ HuAFCs underwent normal cell division. CD44-/CDI05- HuAFCs could not be detected along the injection tract in POF mouse ovaries. Original magnification: $\times 200$. 
CD44-/CD105-
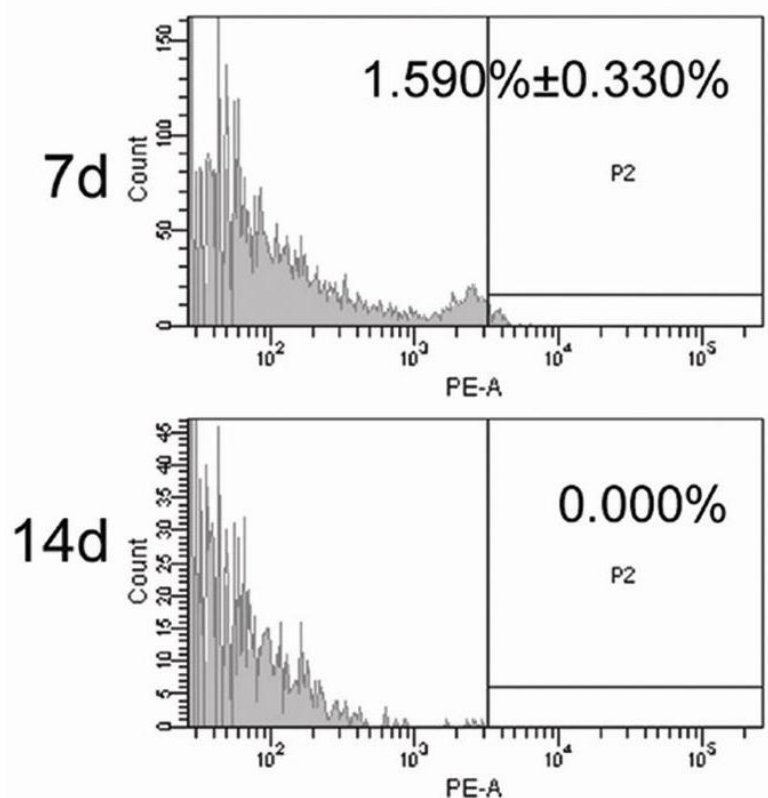

PE-A

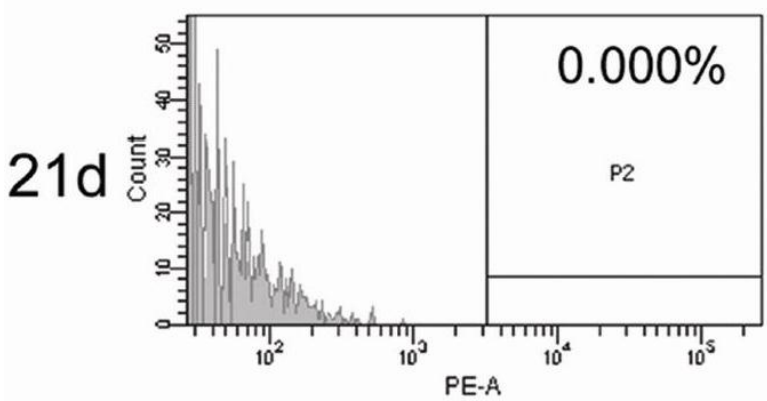

CD44+/CD105+
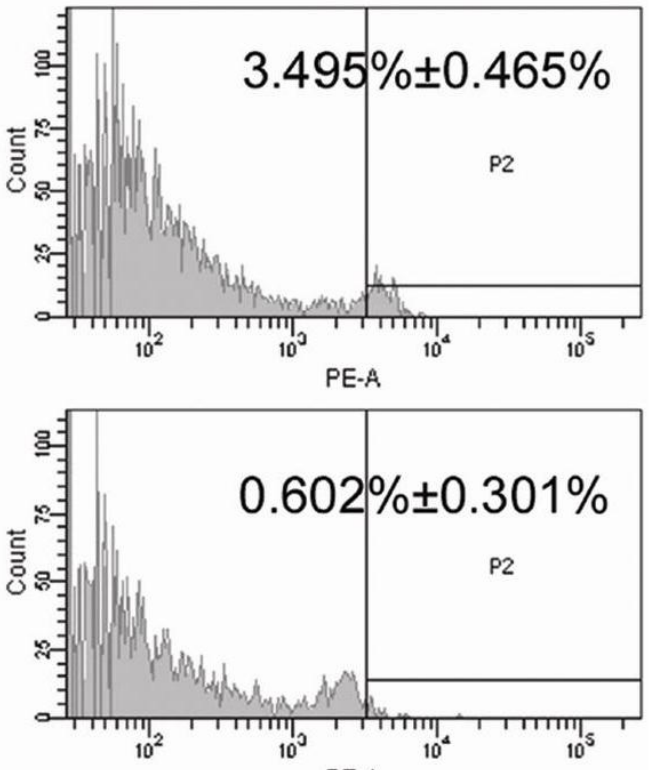

PE-A

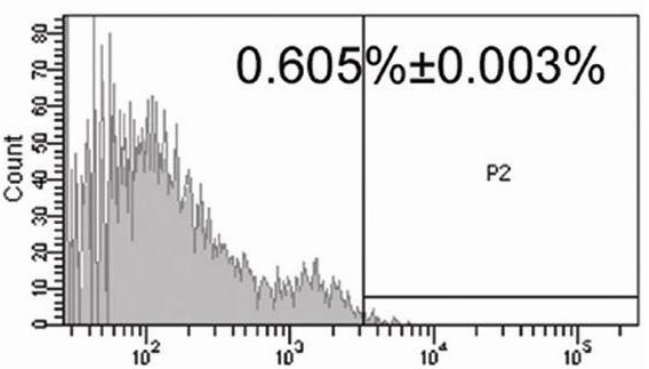

PE-A

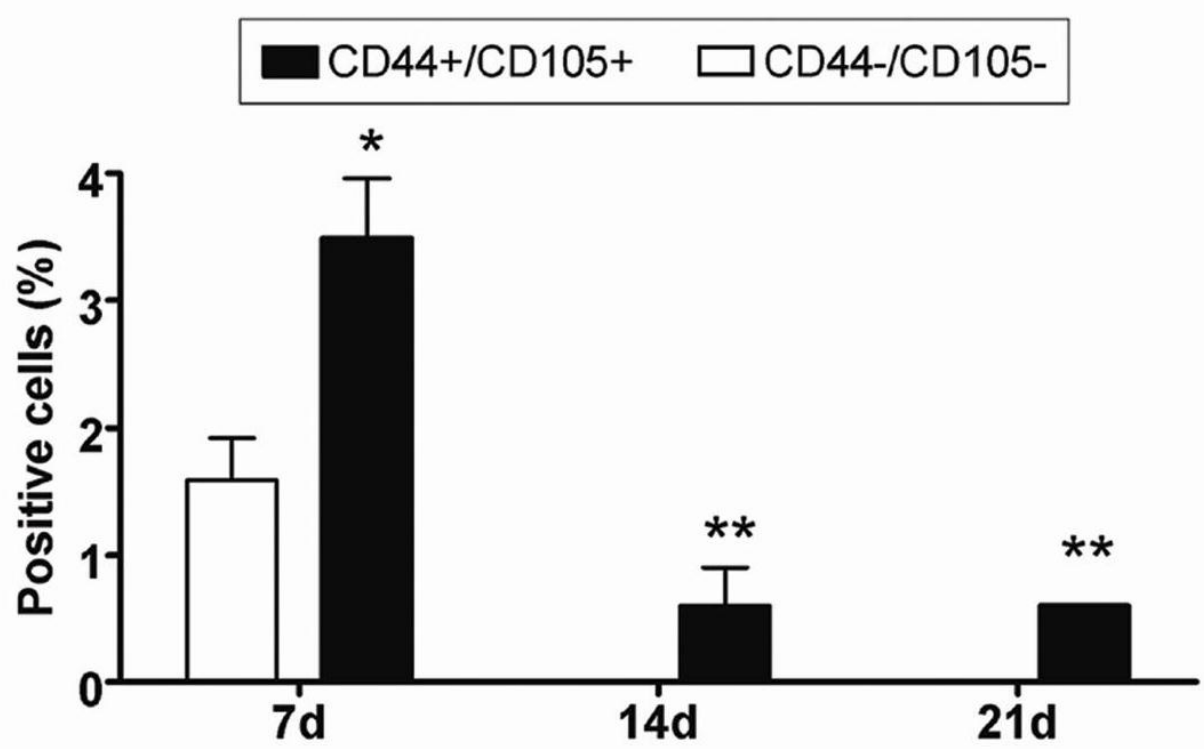

Figure 5. HuAFCs proliferated in vivo in the ovary in a mouse model of POF. Immunofluorescent staining and FCM indicated that the number of Dil positive (red, a marker of HuAFCs) CD44+/CD I05+ HuAFCs increased from the time of the graft up to three weeks after transplantation. RFP-transduced CD44-/CD I05- HuAFCs could not be detected. $* * P<0.01$ vs. CD44-/CDI05- HuAFCs; $* P<0.05$ vs. CD44-/CDI05- HuAFCs; $n=3$. 


\section{Discussion}

Premature ovarian failure (POF), a condition which causes amenorrhoea and hypergonadotropic hypoestrogenism before the age of 40 , affects $1 \%$ of women in the general population $[1,2,7,8]$. The occurrence of POF has increased in recent years. As yet, POF cannot be reversed and though some treatments are currently available, improved treatment strategies are urgently required. Regenerative medicine research suggests that stem cells could be used to treat various human diseases, due to their self-renewal capacity and multiplex differentiation potential. In view of this idea, it is possible that suitable stem cells could be used to treat POF. In our previous studies, we observed that CD44+/CD105+ human amniotic fluid cells (HuAFCs) express a variety of growth factors, lack major histocompatibility complex class II antigens and express only low levels of major histocompatibility complex class I antigens. Furthermore, we previously demonstrated that CD44+/CD105+ HuAFCs can be induced to differentiate into somatic cells [19-21]. Therefore, we hypothesised that CD44+/CD105+ HuAFCs could potentially be used to treat POF. Stem cell transplantation has been reported to rescue ovarian function in a preclinical mouse model of chemotherapy-induced premature ovarian failure (POF) [4]. However, maintaining the survival and self-renewal of seed cells in ovarian tissues over the long-term remains a troublesome issue. In this study, we aimed to determine whether the CD44+/CD105+ HuAFC subpopulation could survive and proliferate in vitro and in vivo over the long-term.

CD44+/CD105+ HuAFCs were successfully enriched from human amniotic fluids using magnetic activated cell sorting. Thereafter, the CD44+/CD105+ and CD44-/CD105- HuAFCs were cultured under the same conditions, and their "stemness" properties and proliferation rates were compared. MTT assays indicated that the viability of CD44-/CD105- HuAFCs significantly reduced on day 3 in vitro, in contrast to CD44+/CD105+ HuAFCs. BrdUrd incorporation and FCM confirmed that CD44+/CD105+ HuAFCs proliferated in vitro, but CD44-/CD105- HuAFCs did not. Western blotting and qRT-PCR showed that the levels of the proliferation markers Ki67 and survivin were significantly higher in CD44+/CD105+ HuAFCs than CD44-/CD105- HuAFCs. Furthermore, the expression levels of a number of "stemness" markers were higher in CD44+/CD105+ HuAFCs than CD44-/CD105HuAFCs. Taken together, these results demonstrated that the CD44+/CD105+ subpopulation possesses more of the properties of mesenchymal stem cells than the CD44-/CD105- subpopulation. Next, the two subpopulations of cells were transplanted into the ovaries of a mouse model of POF. After three weeks, RFP-positive CD44+/CD105+ HuAFCs could be observed along the injection tract in the ovaries of the POF mice, however, RFP-positive CD44-/CD105HuAFCs could not be detected, demonstrating that transplanted CD44+/CD105+ HuAFCs can survive within the POF mouse ovaries for at least three weeks in vivo. Additionally, FCM revealed that the number of CD44+/CD105+ HuAFCs increased appreciably between the time of the graft and three weeks after transplantation, while the RFP-transduced CD44-/CD105- HuAFCs could not be detected. BrdUrd incorporation and FCM confirmed that the transplanted CD44+/CD105+ HuAFCs underwent normal cell division and proliferation.

In conclusion, CD44+/CD105+ HuAFCs possess the characteristics of mesenchymal stem cells, and can survive and proliferate over the long term in the ovarian tissues of a mouse model of chemotherapy-induced POF.

\section{Acknowledgements}

This work was supported by grants from Shanghai Committee Medical Science Foundation of China (No.10411967100) and the Natural Science Foundation of School of Medicine of Shanghai Jiaotong University (No.YZ1046) to Te Liu.

\section{Competing Interests}

The authors have declared that no competing interest exists.

\section{References}

1. Bandyopadhyay S, Chakrabarti J, Banerjee S, Pal AK, Goswami SK, Chakravarty BN, et al. Galactose toxicity in the rat as a model for premature ovarian failure: an experimental approach readdressed. Hum Reprod. 2003; 18: 2031-2038.

2. McGuire MM, Bowden W, Engel NJ, Ahn HW, Kovanci E, Rajkovic A. Genomic analysis using high-resolution single-nucleotide polymorphism arrays reveals novel microdeletions associated with premature ovarian failure. Fertil Steril. 2010; 95: 1595-1600

3. Duncan M, Cummings L, Chada K. Germ cell deficient (gcd) mouse as a model of premature ovarian failure. Biol Reprod. 1993; 49: 221-227.

4. Lee HJ, Selesniemi K, Niikura Y, Niikura T, Klein R, Dombkowski DM, et al. Bone marrow transplantation generates immature oocytes and rescues long-term fertility in a preclinical mouse model of chemotherapy-induced premature ovarian failure. J Clin Oncol. 2007; 25: 3198-3204.

5. Yucebilgin MS, Terek MC, Ozsaran A, Akercan F, Zekioglu O, Isik E, et al. Effect of chemotherapy on primordial follicular reserve of rat: an animal model of premature ovarian failure and infertility. Aust N Z J Obstet Gynaecol. 2004; 44: 6-9.

6. Rebar RW. Premature ovarian "failure" in the adolescent. Ann N Y Acad Sci. 2008; 1135: 138-145.

7. Beck-Peccoz P, Persani L. Premature ovarian failure. Orphanet J Rare Dis. 2006; 1: 9.

8. Persani L, Rossetti R, Cacciatore C. Genes involved in human premature ovarian failure. J Mol Endocrinol. 2010; 45: 257-279. 
9. Prusa AR, Marton E, Rosner M, Bernaschek G, Hengstschlager M. Oct-4-expressing cells in human amniotic fluid: a new source for stem cell research? Hum Reprod. 2003; 18: 1489-1493.

10. Kaviani A, Perry TE, Dzakovic A, Jennings RW, Ziegler MM, Fauza DO. The amniotic fluid as a source of cells for fetal tissue engineering. J Pediatr Surg. 2001; 36: 1662-1665.

11. De Coppi P, Bartsch G, Jr., Siddiqui MM, Xu T, Santos CC, Perin L, et al. Isolation of amniotic stem cell lines with potential for therapy. Nat Biotechnol. 2007; 25: 100-106.

12. Karlmark KR, Freilinger A, Marton E, Rosner M, Lubec G, Hengstschlager M. Activation of ectopic Oct-4 and Rex-1 promoters in human amniotic fluid cells. Int J Mol Med. 2005; 16: 987-992.

13. Lin HT, Chiou SH, Kao CL, Shyr YM, Hsu CJ, Tarng YW, et al. Characterization of pancreatic stem cells derived from adult human pancreas ducts by fluorescence activated cell sorting. World J Gastroenterol. 2006; 12: 4529-4535.

14. Tsai MS, Lee JL, Chang YJ, Hwang SM. Isolation of human multipotent mesenchymal stem cells from second-trimester amniotic fluid using a novel two-stage culture protocol. Hum Reprod. 2004; 19: 1450-1456.

15. Bossolasco P, Montemurro T, Cova L, Zangrossi S, Calzarossa C, Buiatiotis S, et al. Molecular and phenotypic characterization of human amniotic fluid cells and their differentiation potential. Cell Res. 2006; 16: 329-336.

16. In 't Anker PS, Scherjon SA, Kleijburg-van der Keur C, Noort WA, Claas $\mathrm{FH}$, Willemze R, et al. Amniotic fluid as a novel source of mesenchymal stem cells for therapeutic transplantation. Blood. 2003; 102: 1548-1549.

17. Li C, Zhou J, Shi G, Ma Y, Yang Y, Gu J, et al. Pluripotency can be rapidly and efficiently induced in human amniotic fluid-derived cells. Hum Mol Genet. 2009; 18: 4340-4349.

18. Liu T, Xu F, Du X, Lai D, Zhao Y, Huang Q, et al. Establishment and characterization of multi-drug resistant, prostate carcinoma-initiating stem-like cells from human prostate cancer cell lines 22RV1. Mol Cell Biochem. 2010; 340: 265-273.

19. Liu T, Zou G, Gao Y, Zhao X, Wang H, Huang Q, et al. High Efficiency of Reprogramming CD34(+) Cells Derived from Human Amniotic Fluid into Induced Pluripotent Stem Cells with Oct4. Stem Cells Dev. 2012; 21: 2322-2332.

20. Liu T, Guo L, Liu Z, Huang Y, Cheng W. Induction of dopaminergic neuronal-like cells from CD44+ human amniotic fluids that are ameliorative to behavioral recovery in a Parkinson's disease rat model. Int J Mol Med. 2011; 28: 745-752.

21. Zou G, Liu T, Zhang L, Liu Y, Li M, Du X, et al. Induction of pancreatic beta-cell-like cells from CD44+/CD105+ human amniotic fluids via epigenetic regulation of the pancreatic and duodenal homeobox factor 1 promoter. DNA Cell Biol. 2010; 30: 739-748. 